\title{
The preparation of Ni-plated polystyrene microspheres using 3-(trimethoxysilyl) propyl methacrylate as a bridging agent
}

\author{
Wen-Chang Liaw ${ }^{1}$, Yu-Lin Cheng ${ }^{1}$, Ming-Kuen Chang ${ }^{2}$, Wan-Fu Lien ${ }^{3}$ and Hou-Ren Lai ${ }^{1}$
}

This study focuses on the preparation of Ni-plated polystyrene (PS) microspheres (diameter 3.7-4.5 $\mu \mathrm{m}$ ). These Ni-plated PS microspheres have core-shell structures that were designed and prepared using an organic polymer core (PS with 3-(trimethoxysilyl) propyl methacrylate (TSPM)) and an inorganic metal (Ni) plated shell. The polymer microspheres were synthesized by dispersion copolymerization, and the metal shell was then added by electroless plating. To improve the adhesion between the organic polymers and the inorganic metal, a silane bridging agent (TSPM) was used, which was copolymerized with the styrene monomer and modified the PS microsphere surface. Characterization of the Ni-plated PS microspheres was undertaken using Fourier transform infrared spectroscopy (FT-IR), thermo-gravimetric analysis (TGA), X-ray diffraction (XRD), field emission scanning electron microscopy (FE-SEM) and the ball mill. The results showed that the Ni-plated PS microspheres were successfully prepared. The PS microspheres copolymerized with the silane bridging agent contain more binding sites for $\mathrm{Ni}$, and the bonds formed with $\mathrm{Ni}$ are stable. The distribution of Ni-plating on the PS microspheres is more uniform and denser in the presence of TSPM. In ball mill, the Ni-plated PS microspheres containing silane bridges were more durable than those without silane bridges.

Polymer Journal (2016) 48, 91-99; doi:10.1038/pj.2015.88; published online 7 October 2015

\section{INTRODUCTION}

Recently, monodisperse metal-plated polymer microspheres have received much attention owing to their high conductivity and low density. They combine the properties of both metal and polymer. Metal-plated polymer microspheres have core-shell structures with potential applications in biosensors, conductive particles, microelectronic devices and electronic packaging. ${ }^{1-5}$ In the literature, various approaches have been exploited to produce uniformly sized polymer core microsphere colloids: these approaches include suspension polymerization, ${ }^{6,7}$ emulsion polymerization ${ }^{8}$ and dispersion polymerization. ${ }^{9-11}$ Several polymer microsphere substrates have been used in electroless plating; these include polystyrene (PS) ${ }^{12-14}$ and polymethylmethacrylate (PMMA) microspheres, ${ }^{15,16}$ as well as polyaniline powders ${ }^{17}$ and acrylate rubber. ${ }^{18}$

The main problem in the preparation of metal-plated polymer microspheres is the weak adhesion between the metal layer and the polymer substrate. The best resolution of this problem is to copolymerize the monomer with an agent containing organic functional groups, which modify the polymer core surface. This modification can increase the affinity and reduce costs in during electroless plating. The organic functional groups readily adsorb the plating metal ions. Many functional groups have been studied, such as amino, ${ }^{19}$ carboxyl, ${ }^{20}$ sulfonic acid ${ }^{21}$ and silane groups. ${ }^{22,23}$

Polysiloxane (a silane bridging agent) has many useful properties, such as excellent thermal stability, weather resistance, lubricity and good water repellency. ${ }^{24-26}$ For example, 3-(trimethoxysilyl) propyl methacrylate (TSPM) with methoxysilyl groups $\left[\mathrm{Si}\left(\mathrm{OCH}_{3}\right)_{3}\right]$ has been widely used in the preparation of organic/inorganic hybrid materials. ${ }^{27-29}$ The silane bridging agent has a trialkoxysilyl group, which may react with hydroxyl groups on the metal surface and then form Si-O-metal covalent bonds. ${ }^{30-32}$ The acrylic functional group of the silane bridging agent was first cross-linked with styrene monomer, and the trialkoxysilyl or trihydroxysilyl functional groups of the silane bridging agent were then exposed on the surface of the PS microspheres to form Si-O-metal covalent bonds during the metal deposition.

In the synthesis procedure, the silane bridging agent TSPM copolymerized with the styrene monomer; then, the silane bridging agent migrated to the surface of the microspheres, as some of the silane bridging agent was grafted onto the surface of the microspheres by reaction with PS to form covalent bonds. ${ }^{33}$ In the electroless plating stage, the surfaces of these microspheres have trihydroxysilyl

\footnotetext{
${ }^{1}$ Department of Chemical and Materials Engineering, National Yunlin University of Science and Technology, Yunlin, Taiwan; ${ }^{2}$ Department of Safety, Health and Environmental Engineering, National Yunlin University of Science and Technology, Yunlin, Taiwan and ${ }^{3}$ Department of Cultural Heritage Conservation, National Yunlin University of Science and Technology, Yunlin, Taiwan

Correspondence: Professor M-K Chang, Department of Safety, Health and Environmental Engineering, National Yunlin University of Science and Technology, \#123, Section 3, University Road, Touliu, Yunlin 64002, Taiwan.

E-mail: changmk@yuntech.edu.tw

Received 20 May 2015; revised 31 July 2015; accepted 13 August 2015; published online 7 October 2015
} 
functional groups, which can improve adhesion between the organic polymer and the metal as a result of the formation of the Si-O-metal covalent bonds mentioned above. The silane bridging agent is bound to the polymer core and inorganic metal shell, and it increases the number of sites where a stable metal bridge can form.

In this study, a series of PS microspheres was prepared by the dispersion copolymerization of styrene (St) and TSPM. The PS microspheres, which include the same PS core and different thicknesses of the polysiloxane surface, can have different particle sizes and thermal stabilities. The sizes of the PS microspheres were controlled in the range of $3.5-4.7 \mu \mathrm{m}$. The $\mathrm{Ni}$ was then sequentially deposited on these PS microspheres using electroless plating. The main purpose of this study was to prepare Ni-plated PS microspheres by using a silane bridging agent to form covalent bonds between the PS microspheres surface and the Ni layer. The polymer microsphere surfaces have

Table 1 Composition of the core-shell structure of Ni-plated PS microspheres

\begin{tabular}{|c|c|c|c|}
\hline \multicolumn{2}{|c|}{ Sample code } & \multirow[t]{2}{*}{$T S P M(g)$} & \multirow[t]{2}{*}{ TSPM (wt\%) } \\
\hline Before Ni-plating & After Ni-plating & & \\
\hline PS & $\mathrm{PS} / \mathrm{Ni}$ & 0 & 0 \\
\hline PSS1 & PSS1/Ni & 0.18 & 0.3 \\
\hline PSS2 & PSS2/Ni & 0.3 & 0.5 \\
\hline PSS3 & PSS3/Ni & 0.45 & 0.75 \\
\hline PSS4 & PSS4/Ni & 0.6 & 1 \\
\hline
\end{tabular}

Abbreviations: AIBN, 2,2-azobisisobutyronitrile; PS, polystyrene; PVP, polyvinyl pyrrolidone; TSPM, 3-(trimethoxysilyl) propyl methacrylate. The styrene (60 g), PVP K30 (2.4 g), AIBN (2 g) and different TSPM were homogeneously dissolved in the ethanol $(140 \mathrm{~g})$. trialkoxysilyl groups and trihydroxysilyl functional groups, and the formation of the Si-O-Ni covalent bond can improve the adhesion between the interface of the organic polymer microspheres and the nickel metal. The products of Ni-plated PS microspheres were characterized using Fourier transform infrared spectroscopy (FT-IR), thermo-gravimetric analysis (TGA), field emission scanning electron microscopy (FE-SEM), X-ray diffraction (XRD) and the ball mill.

\section{EXPERIMENTAL PROCEDURE}

\section{Materials}

St, TSPM, succinic acid disodium salt $\left(\mathrm{C}_{4} \mathrm{H}_{4} \mathrm{Na}_{2} \mathrm{O}_{4}\right)$ and palladium chloride $\left(\mathrm{PdCl}_{2}\right)$ were sourced from Sigma-Aldrich (St Louis, MO, USA). 2,2-Azobisisobutyronitrile (AIBN, initiator) was purchased from SHOWA Chemical Co. Ltd (Tokyo, Japan). Polyvinyl pyrrolidone (PVP K30 Mw $=40$ 000, stabilizer) was purchased from First Chemical Co. Ltd (Taipei, Taiwan). Ethanol was purchased from Uni-onward Co. Ltd (95\%, Taipei, Taiwan). Stannous chloride dihydrate $\left(\mathrm{SnCl}_{2} \cdot 2 \mathrm{H}_{2} \mathrm{O}\right)$, nickel chloride hexahydrate $\left(\mathrm{NiCl}_{2} \cdot 6 \mathrm{H}_{2} \mathrm{O}\right)$ and sodium hypophosphite $\left(\mathrm{NaH}_{2} \mathrm{PO}_{2} \cdot \mathrm{H}_{2} \mathrm{O}\right)$ were obtained from Shimakyu Chemical Co. Ltd (Osaka, Japan). Deionized water was used in all experiments.

\section{Preparation of functionalized PS microspheres}

Functionalized PS microspheres were prepared by dispersion copolymerization using St, TSPM, AIBN and PVP. The polymerization was conducted in a 500-ml five-neck round-bottom flask equipped with a stirrer, reflux condenser, thermometer, nitrogen inlet tube and feeding funnel. In the initial stage, St, TSPM, AIBN and PVP were homogeneously dissolved in the ethanol. The polymerization temperature was fixed at $70^{\circ} \mathrm{C}$, and the speed of the stirrer was 250 r.p.m. in a nitrogen atmosphere. The polymerization proceeded for $8 \mathrm{~h}$. The resultant product was filtered with a Buchner funnel, and the filter cake was washed with ethanol and then with deionized water three times. Finally, the PS microspheres that had been copolymerized with TSPM were dried in an oven at $50^{\circ} \mathrm{C}$ for $24 \mathrm{~h}$. All of the ingredients used are summarized in Table 1 .

\section{Reaction 1}
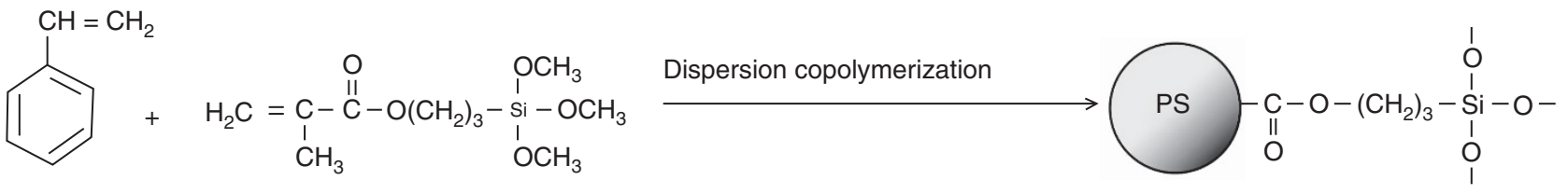

Styrene

3-(trimethoxysilyl) propyl methacrylate

\section{Reaction 2}
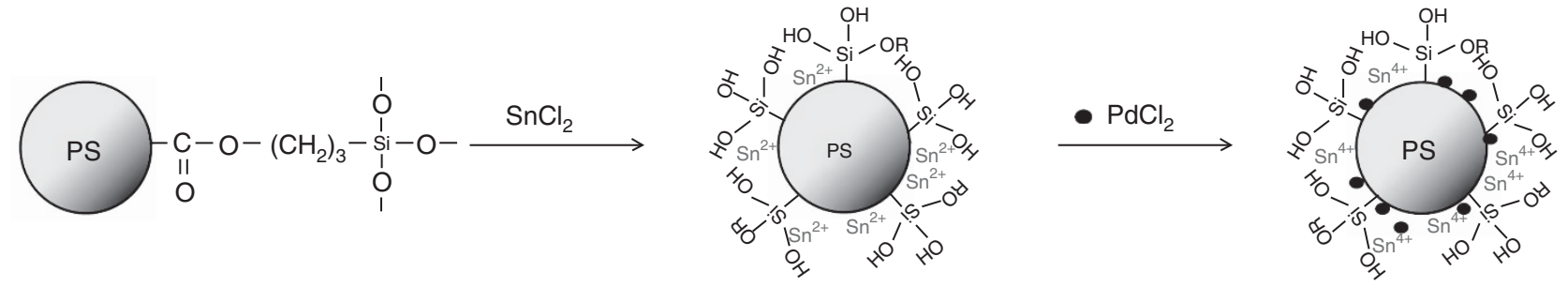

- Pd nanoparticle

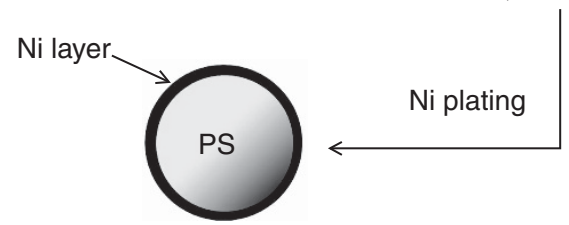

Scheme 1 Reaction diagram for electroless nickel plating on functionalized PS microspheres. A full color version of this figure is available at Polymer Journal online. 
Electroless nickel plating on functionalized PS microspheres

Nickel was coated onto the functionalized PS microspheres by electroless plating. The procedure is illustrated in Scheme 1. The functionalized PS microspheres were sensitized using an acidic $\mathrm{SnCl}_{2}$ solution $\left(0.044 \mathrm{moll}^{-1}\right.$ $\mathrm{SnCl}_{2}$ and $0.22 \mathrm{moll}^{-1} \mathrm{HCl}$ ) for $30 \mathrm{~min}$ at $45^{\circ} \mathrm{C}$, resulting in the adsorption of $\mathrm{Sn}^{2+}$ ions onto the surface of the PS microspheres. Then, the PS microspheres were centrifuged to remove unabsorbed $\mathrm{Sn}^{2+}$ and washed with deionized water. Next, the $\mathrm{Sn}^{2+}$-functionalized PS microspheres were activated using an acidic $\mathrm{PdCl}_{2}$ solution $\left(5.6 \times 10^{-3} \mathrm{moll}^{-1} \mathrm{PdCl}_{2}\right.$ and $\left.0.22 \mathrm{moll}^{-1} \mathrm{HCl}\right)$ for $1 \mathrm{~h}$ at $75^{\circ}$ C. A surface redox reaction occurred in this step, which involved the reduction of $\mathrm{Pd}^{2+}$ to $\mathrm{Pd}$ and the oxidation of $\mathrm{Sn}^{2+}$ to $\mathrm{Sn}^{4+}$. In this step, metallic $\mathrm{Pd}$, serving as the catalytic sites, was formed and was chemically bonded to the functional groups (trialkoxysilyl and trihydroxysilyl) on the functionalized surface of the PS microspheres. After removal of the supernatant by centrifugation, the activated functionalized PS microspheres were washed with distilled water.

Ni-plated PS microspheres (PS/Ni) were prepared by immersing the pretreated microspheres in a nickel-plating solution at $90^{\circ} \mathrm{C}$ to produce an Ni layer under constant stirring. The compositions of the nickel-plating solution are provided in Table 2. Mechanical agitation was employed during this electroless plating process. After $30 \mathrm{~min}$, the gray-black powders were filtered and washed with distilled water, then dried at $50^{\circ} \mathrm{C}$ for $24 \mathrm{~h}$.

\section{Characterization}

The functional groups of the microspheres were observed by FT-IR (Perkin-Elmer, Spectrum One) (Waltham, MA, USA), within the range $450-4000 \mathrm{~cm}^{-1}$. The thermal degradation and thermal stability of the

Table 2 Compositions of the solutions for sensitization, activation and electroless plating

\begin{tabular}{lcc}
\hline Process & Chemicals & Concentration $\left(\mathrm{mol}^{-1}\right)$ \\
\hline Sensitization & $\mathrm{SnCl}_{2} \cdot 2 \mathrm{H}_{2} \mathrm{O}$ & 0.044 \\
Activation & $\mathrm{HCl}$ & 0.22 \\
& $\mathrm{PdCl}_{2}$ & $5.6 \times 10^{-3}$ \\
Electroless Ni-plating & $\mathrm{HCl}$ & 0.22 \\
& $\mathrm{NiCl}_{2} \cdot 6 \mathrm{H}_{2} \mathrm{O}$ & 0.0841 \\
& $\mathrm{NaH}_{2} \mathrm{PO}_{2} \cdot \mathrm{H}_{2} \mathrm{O}$ & 0.217 \\
& $\mathrm{C}_{4} \mathrm{H}_{4} \mathrm{Na}_{2} \mathrm{O}_{4}$ & 0.0987 \\
\hline
\end{tabular}

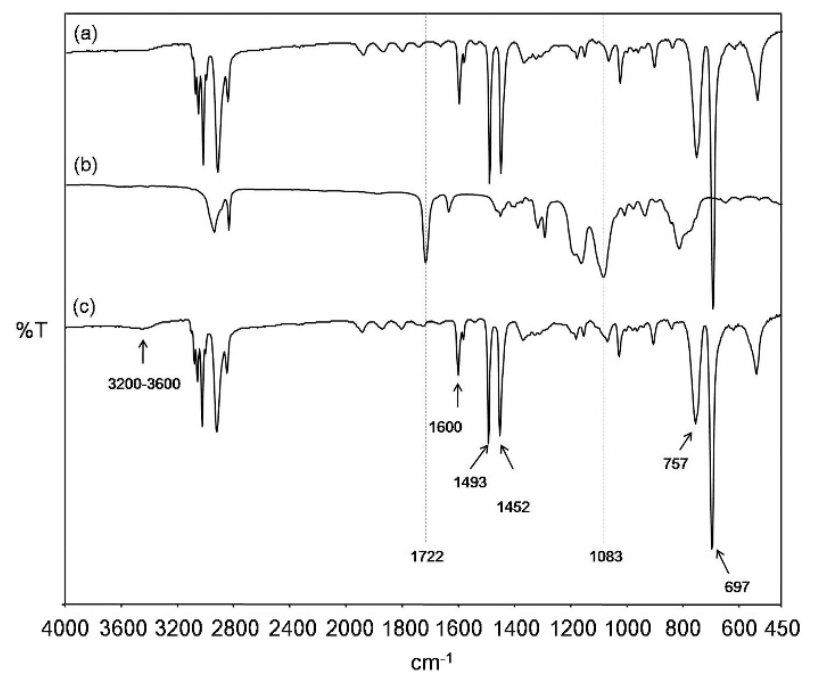

Figure 1 FT-IR spectra of (a) PS, (b) TSPM and (c) PSS4. microspheres were monitored by TGA (TA Instruments Inc., Q500) (New Castle, DE, USA). The TGA measurements were performed under a nitrogen atmosphere at a heating rate of $20^{\circ} \mathrm{C}$ per minute, from 25 to $800^{\circ} \mathrm{C}$. Micrographs of the PS microspheres before and after TSPM functionalization, as well as after nickel plating, were taken by FE-SEM (Jeol Ltd, JSF 6701F) (Tokyo, Japan), which also provides energy-dispersion X-ray analysis (EDX). The elements of the PS microspheres before and after nickel plating were identified by XRD patterns at a scanning rate of $5^{\circ}$ per minute within the range of $2 \theta=20-80^{\circ}$ (Bruker, P4 single crystal X-ray diffractometer) (Rheinstetten, Germany). The adhesion strength between the coated metal layers and the microspheres was explored through a ball mill (Shin Kwang Machinery Industry, UBM-1 ball mill) (Taipei, Taiwan) for $30 \mathrm{~min}$ at a rate of 150 r.p. $\mathrm{m}$. The volume of the ball mill was $250 \mathrm{ml}$. The average diameter of the balls used for milling was $1.62 \mathrm{~mm}$. The ball mill loading included $100 \mathrm{~g}$ balls and $2 \mathrm{~g}$ Ni-plated PS microspheres in $10 \mathrm{ml}$ water. After the ball mill, the milled Si-O-Ni surface was also characterized using FE-SEM.

\section{RESULTS AND DISCUSSION}

\section{FT-IR characterization}

FT-IR revealed chemical changes to the microspheres during synthesis. The method was used to verify the presence of functional groups. Figure 1 compares the spectra for PS, TSPM and PSS4. The PS microspheres show the characteristic absorption peaks for the benzene ring at 1600,1493 and $1452 \mathrm{~cm}^{-1}$, the $\mathrm{C}-\mathrm{H}$ stretching of the phenyl ring absorption peaks in the range $3100-3000 \mathrm{~cm}^{-1}$, and the $\mathrm{C}-\mathrm{H}$ bending absorption peaks at 757 and $697 \mathrm{~cm}^{-1} .34$ These absorption peaks confirmed the phenyl ring (styrene) as the main component of the microspheres.

The FT-IR spectra of the TSPM and PSS4 microspheres show the characteristic broad absorption peaks of the trihydroxysilyl group $\left(\mathrm{Si}(\mathrm{OH})_{3}\right)$ at $3200-3600 \mathrm{~cm}^{-1}$, the trialkoxysilyl group at $1083 \mathrm{~cm}^{-1}$ $\left(\mathrm{SiOCH}_{3}\right)$ and the carbonyl group $(\mathrm{C}=\mathrm{O})$ at $1722 \mathrm{~cm}^{-1} .35$ This result confirms that the PS microspheres carrying alkoxysilane groups were synthesized in the dispersion copolymerization using a silane bridging agent (TSPM) as a functional comonomer. The hydrolysis of the trialkoxysilyl group in the copolymerization solution was transferred to the trihydroxysilyl groups upon the copolymerization reaction between the styrene monomer and the silane bridging agent (TSPM). The resultant $\mathrm{Si}-\mathrm{OH}$ function groups paved the way for subsequent covalent bonding (Si-O-Ni). All of these results suggest that some silane bridging agent bound to the PS microsphere surface. The SEM results discussed below confirm that the silane bridging agent migrates

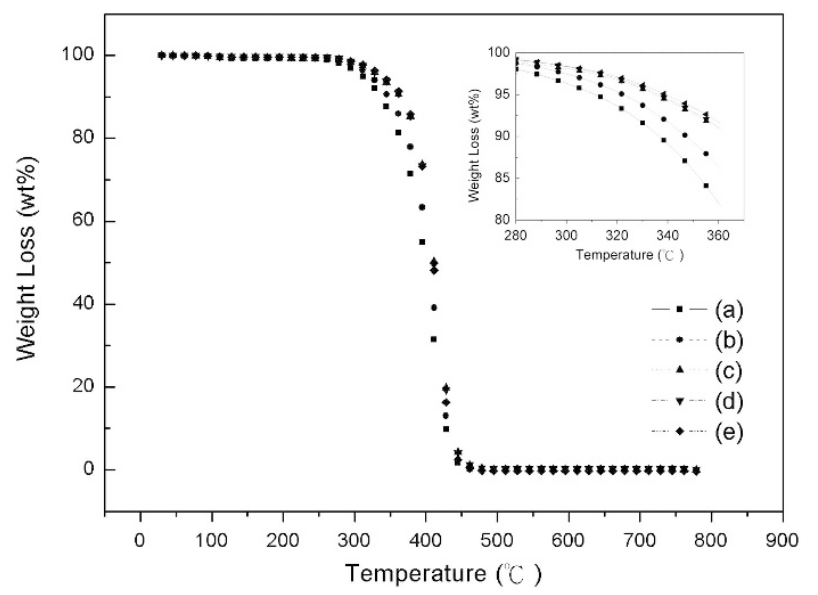

Figure 2 TGA curves of (a) PS, (b) PSS1, (c) PSS2, (d) PSS3 and (e) PSS4. 


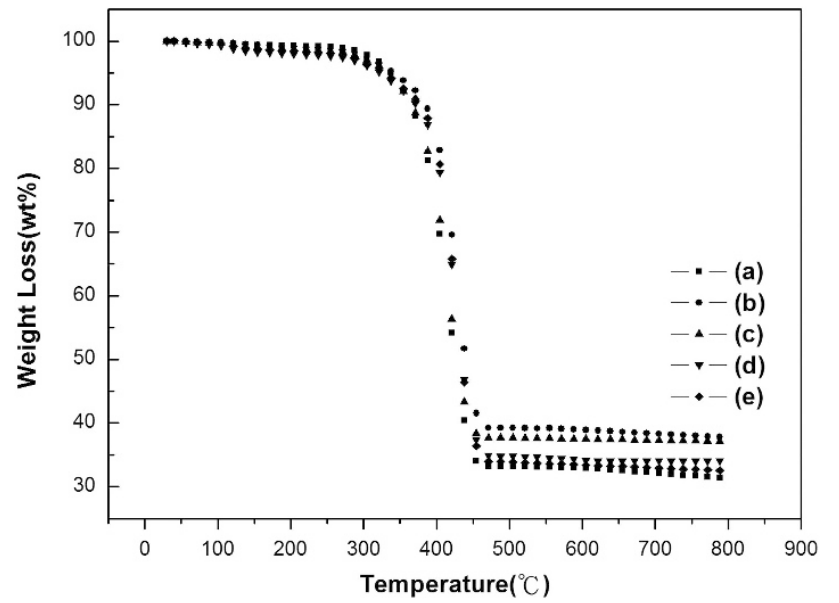

Figure 3 TGA curves of (a) PS/Ni, (b) PSS1/Ni, (c) PSS2/Ni, (d) PSS3/Ni and (e) PSS4/Ni.


Figure 4 SEM images of (a) PS, (b) PSS1, (c) PSS2, (d) PSS3 and (e) PSS4.

to the surface of the microspheres and results in increased nickel plating.

\section{TGA characterization}

The thermal degradation temperatures (Td, at 5\% weight loss) of the microsphere composites were studied by TGA. This study focused on the thermal degradation temperatures of PS microspheres prepared using different concentrations of TSPM. Figure 2 shows that (a) PS, (b) PSS1, (c) PSS2, (d) PSS3 and (e) PSS4 had thermal degradation temperatures of $311,322,333,335$ and $339^{\circ} \mathrm{C}$, respectively. This trend is related to the amount of TSPM, as the C-Si-O and Si-O-Si network structures were formed by condensation on the surface of the polymer core. The thermal stability of PS microspheres depends on the formation intensity of the PS microspheres and the covalent bonds between $\mathrm{C}, \mathrm{O}$ and $\mathrm{Si}$. The bond dissociation energy of the $\mathrm{C}-\mathrm{C}$ bond is lower than that of the C-Si and Si-O bonds. On this basis, we prepared the PS microspheres with different TSPM concentrations. The thermal degradation temperature increased with TSPM content. The silane
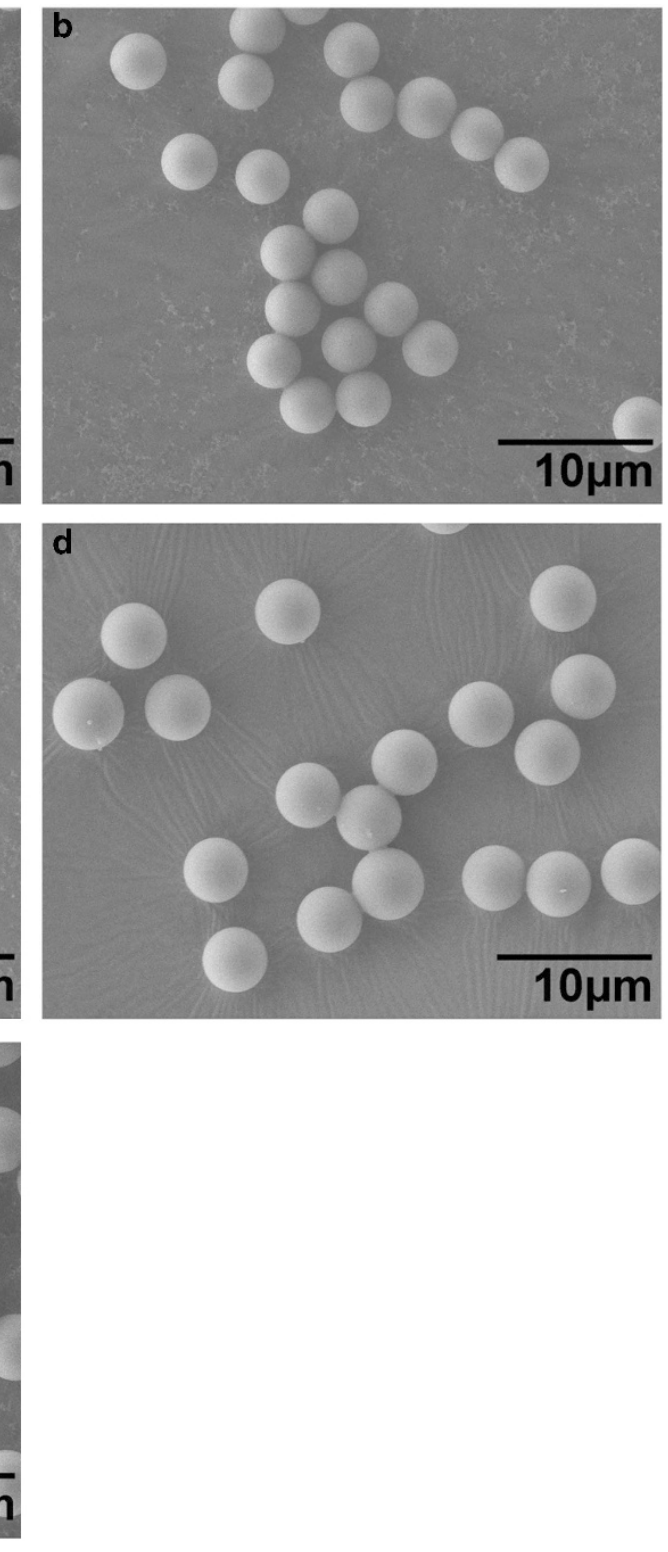
bridging agent cross-linked network structure can thus enhance the thermal stability compared with bare PS microspheres.

The effect of the PS microsphere particle sizes on the Ni-plating content was studied. Figure 3 shows that (a) PS/Ni, (b) PSS $1 / \mathrm{Ni}$, (c) $\mathrm{PSS} 2 / \mathrm{Ni}$, (d) $\mathrm{PSS} 3 / \mathrm{Ni}$ and (e) PSS $/ \mathrm{Ni}$ had weight residues of $31.36,38.14,37.12,35.5$ and $34.02 \mathrm{wt} \%$, respectively. Clearly, the nickel weight residues of PS microspheres modified with TSPM are larger than for the PS microspheres with $0 \mathrm{wt} \%$ TSPM. The particle size of the PS microsphere increased with the TSPM content (0.3-1 wt \%). However, the nickel weight residue ( $w \mathrm{t} \%$ ) of the PS microsphere decreased as the particle size increased, as the smaller PS microsphere particles had larger surface areas.

\section{SEM characterization}

FE-SEM was used to observe the morphology and the microscopic structure of the microspheres. Figure 4 shows SEM images of the PS microspheres synthesized with various concentrations of TSPM. The average particle sizes of (a) PS, (b) PSS1, (c) PSS2, (d) PSS3 and (e) PSS4 increased from 3.421 to $4.158 \mu \mathrm{m}$ (average of all five types). The TSPM concentration influences both the average particle size and the uniformity. Dispersion polymerization is more sensitive than the other synthesis methods. The uniformity of the final particle will change significantly with slight variations in the polymerization ingredients. The acrylic structure of TSPM increased the solvency of the homogeneous reaction mixture, resulting in an increase in particle sizes. The copolymer is more soluble in the reaction medium than is PS, resulting in larger particle sizes. ${ }^{36}$ In this study, all of the particles have retain their spherical shape with TSPM feed ratio increases. It is clear that the silane bridging agent is bound to the PS microspheres in a uniform manner, and the surface of these microspheres is very smooth. As expected, the alkoxysilane of the polysiloxane layer surface of the microspheres has an anchoring function.

Figures 5a-e show the SEM images of PS microspheres containing different concentrations of TSPM after electroless nickel plating.
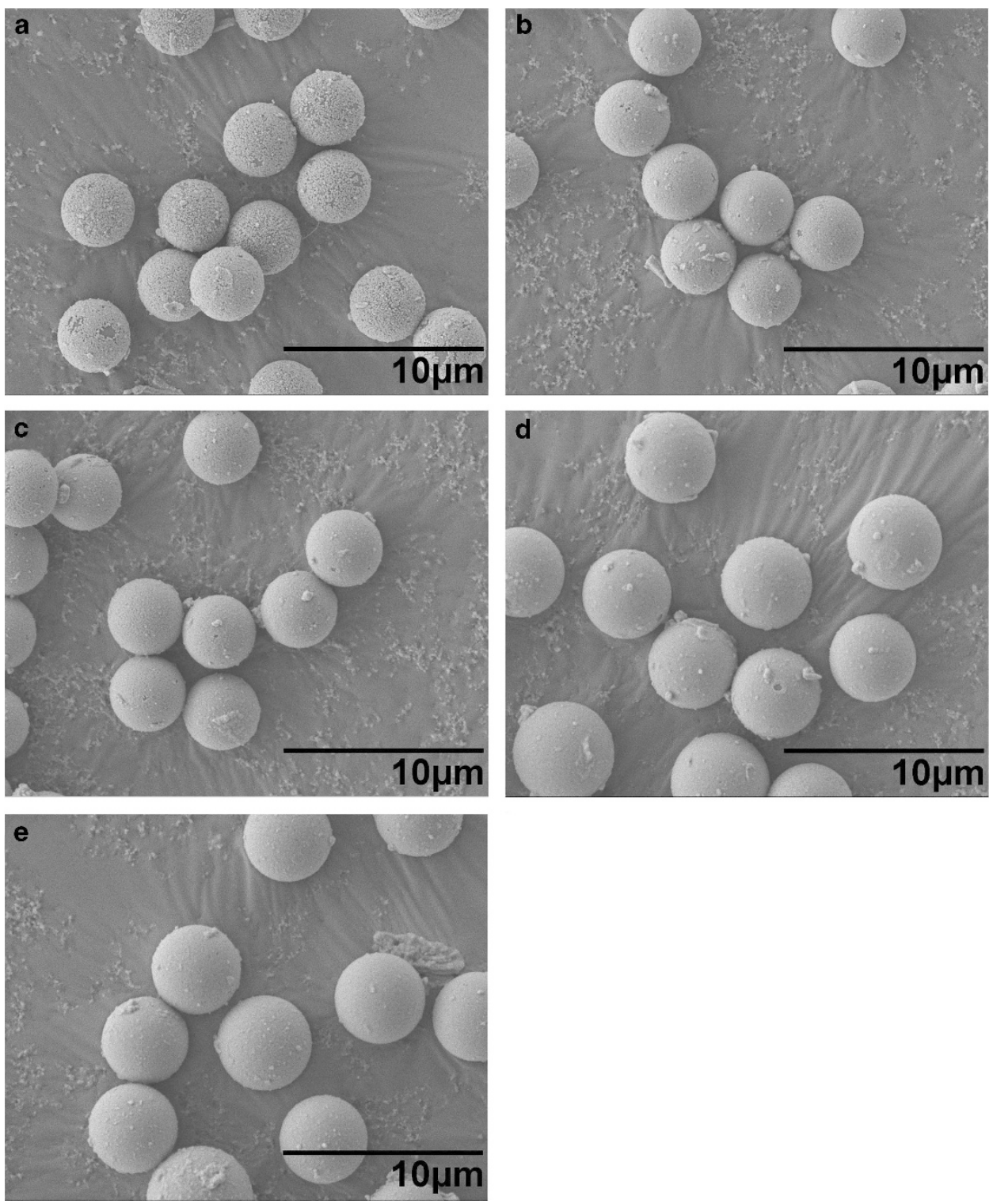

Figure 5 SEM images of (a) PS/Ni, (b) PSS1/Ni, (c) PSS2/Ni, (d) PSS3/Ni and (e) PSS4/Ni. 
The surface patchiness observed in the background is likely to be due to the carbon conductive tape or free nickel particles. Comparing these SEM images, the deposited nickel clearly makes the PS microspheres rougher. In Figure $5 \mathrm{a}(\mathrm{PS} / \mathrm{Ni})$, there

Table 3 Particle sizes before and after electroless Ni-plating

\begin{tabular}{lcccc}
\hline \multicolumn{2}{c}{ Sample code } & & \multicolumn{2}{c}{ Particle size $(\mu \mathrm{m})$} \\
\cline { 5 - 5 } Before Ni-plating & After Ni-plating & & Before Ni-plating & After Ni-plating \\
\hline PS & PS/Ni & & 3.421 & 3.710 \\
PS1 & PSS1/Ni & & 3.474 & 3.790 \\
PS2 & PSS2/Ni & & 3.552 & 3.871 \\
PSS3 & PSS3/Ni & & 4.079 & 4.451 \\
PSS4 & PSS4/Ni & & 4.158 & 4.548 \\
\hline
\end{tabular}

Abbreviations: FE-SEM, field emission scanning electron microscopy; PS, polystyrene. The average diameters of particles are measured by FE-SEM.
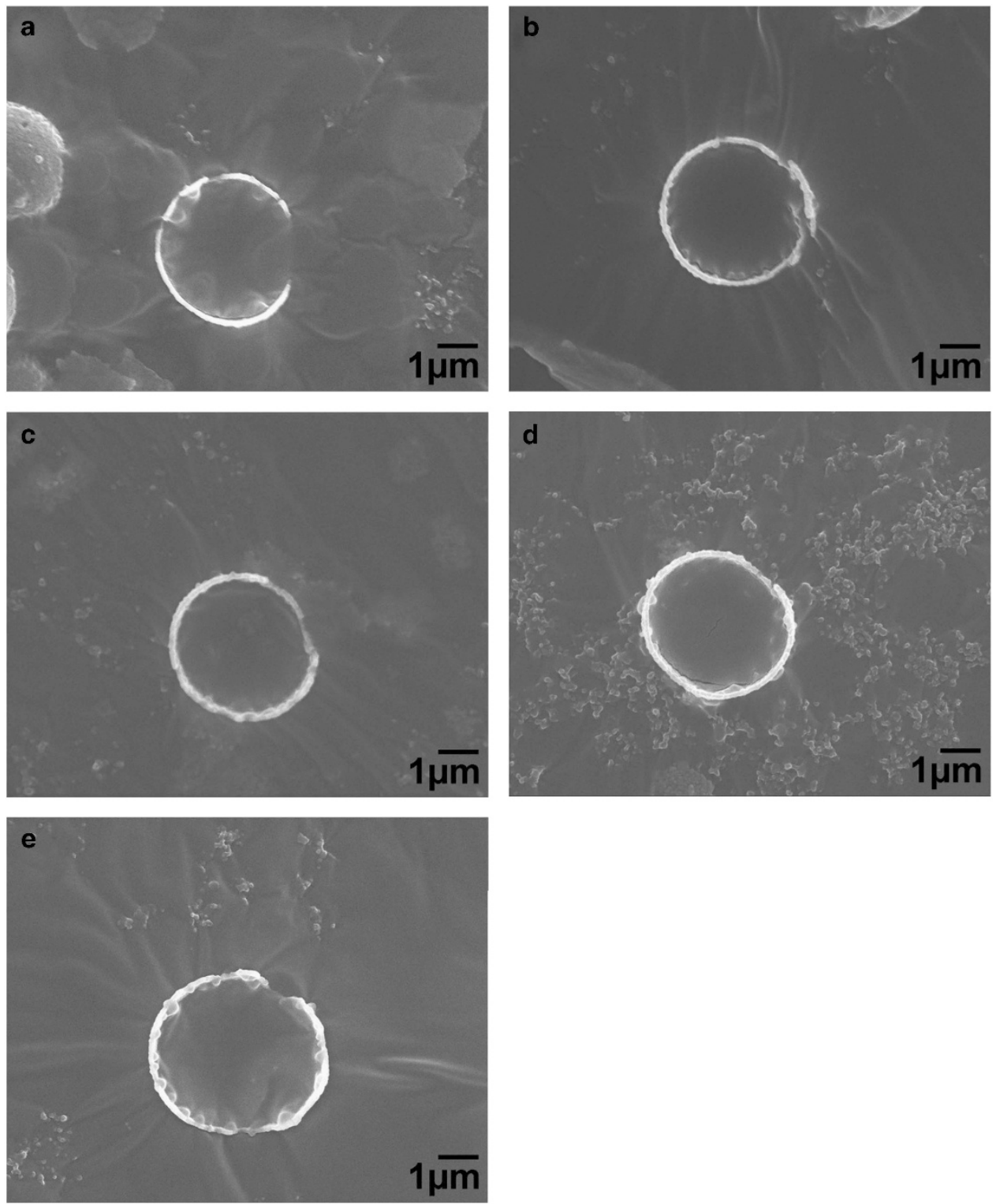

Figure 6 SEM images of (a) PS/Ni, (b) PSS1/Ni, (c) PSS2/Ni, (d) PSS3/Ni and (e) PSS4/Ni after extraction using tetrahydrofuran (THF).

were few nickel aggregate particles on the surface of the PS microspheres containing $0 \mathrm{wt} \%$ TSPM. The discontinuous nickel particles on the surface of PS/Ni reveal that the deposited nickel particles are insufficient to cover the whole surface of the PS

Table $4 \mathrm{Ni}$ shell thickness obtained by different methods.

\begin{tabular}{lccc}
\hline \multicolumn{2}{c}{ Sample code } & $\begin{array}{c}\text { Ni shell } \\
\text { thickness }(\mu \mathrm{m})^{\mathrm{a}}\end{array}$ & $\begin{array}{c}\text { Ni shell } \\
\text { thickness }(\mu \mathrm{m})^{\mathrm{b}}\end{array}$ \\
\hline Before Ni-plating & After Ni-plating & & \\
\hline PS & $\mathrm{PS} / \mathrm{Ni}$ & 0.145 & 0.132 \\
PS1 & $\mathrm{PSS} 1 / \mathrm{Ni}$ & 0.158 & 0.152 \\
PS2 & $\mathrm{PSS} / \mathrm{Ni}$ & 0.160 & 0.165 \\
PSS3 & $\mathrm{PSS} / \mathrm{Ni}$ & 0.186 & 0.195 \\
PSS4 & $\mathrm{PSS} 4 / \mathrm{Ni}$ & 0.195 & 0.217
\end{tabular}

Abbreviations: PS, polystyrene; SEM, scanning electron microscopy.

${ }^{a}$ Calculated from the particle sizes before and after electroless Ni-plating in Table 3. bMeasured from SEM photographs of cross-section results in Figure 6. 


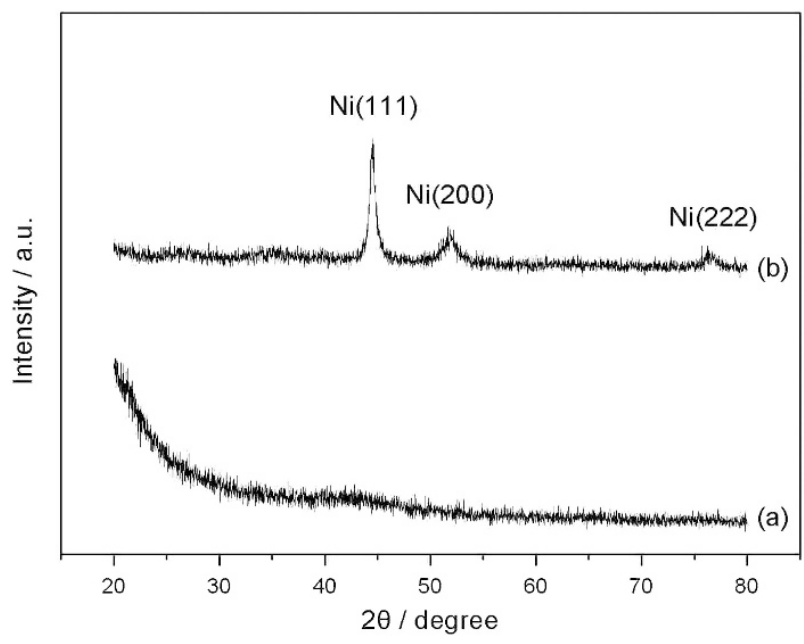

Figure 7 XRD patterns of (a) PSS1 and (b) PSS1/Ni microspheres. microspheres. Table 3 shows the particle sizes before and after electroless Ni-plating.

Figures $5 \mathrm{~b}(\mathrm{PSS} 1 / \mathrm{Ni})$ and $\mathrm{c}(\mathrm{PSS} 2 / \mathrm{Ni})$ show that the nickel plating on the PS microspheres gradually increased and became more uniform in the TSPM concentration range of $0.3-0.5 \mathrm{wt} \%$. Moreover, Figures $5 \mathrm{~d}$ and e show the surfaces of $\mathrm{PSS} 3 / \mathrm{Ni}$ and $\mathrm{PSS} / \mathrm{Ni}$, respectively. The nickel on the PSS3/Ni and PSS4/Ni microspheres was more uniform than on the PS/Ni, PSS1/Ni and PSS2/Ni microspheres when the TSPM concentration increased from 0.75 to $1 \mathrm{wt} \%$. Therefore, it can be concluded that the uniformity of nickel distribution on PS microspheres surfaces is proportional to the TSPM concentration.

Hollow nickel particles were prepared through a core extraction process using tetrahydrofuran. The PS cores were removed by exposure to the solvent. The hollow nickel particles were placed on the carbon conductive tape, and the cross-sections of broken hollow nickel particles were searched for and observed by FE-SEM analysis. The thickness of the nickel plating layer can be obtained for PS/nickel
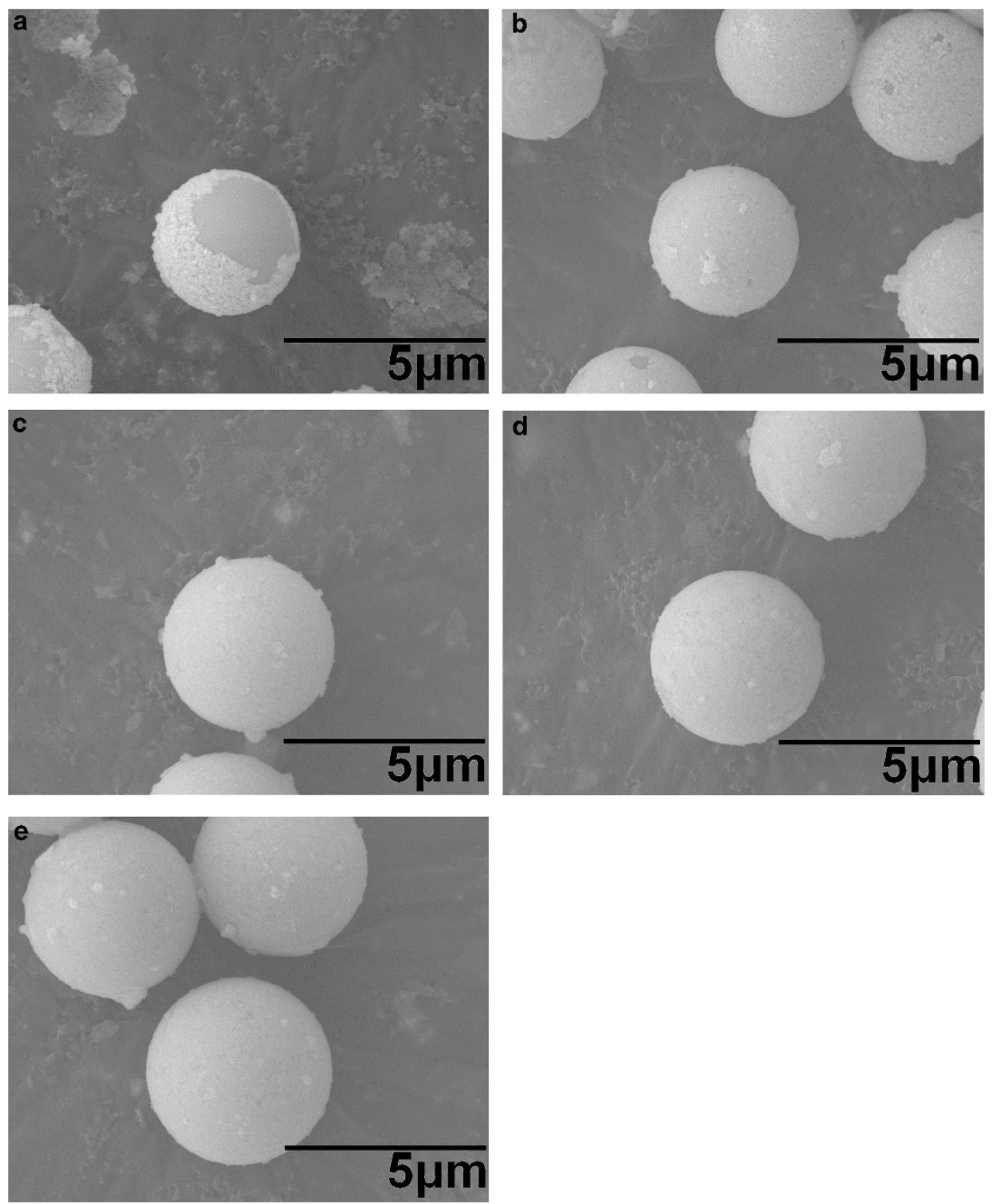

Figure 8 SEM images of (a) PS/Ni, (b) PSS1/Ni, (c) PSS2/Ni, (d) PSS3/Ni and (e) PSS4/Ni after ball mill durability test for $0.5 \mathrm{~h}$. 

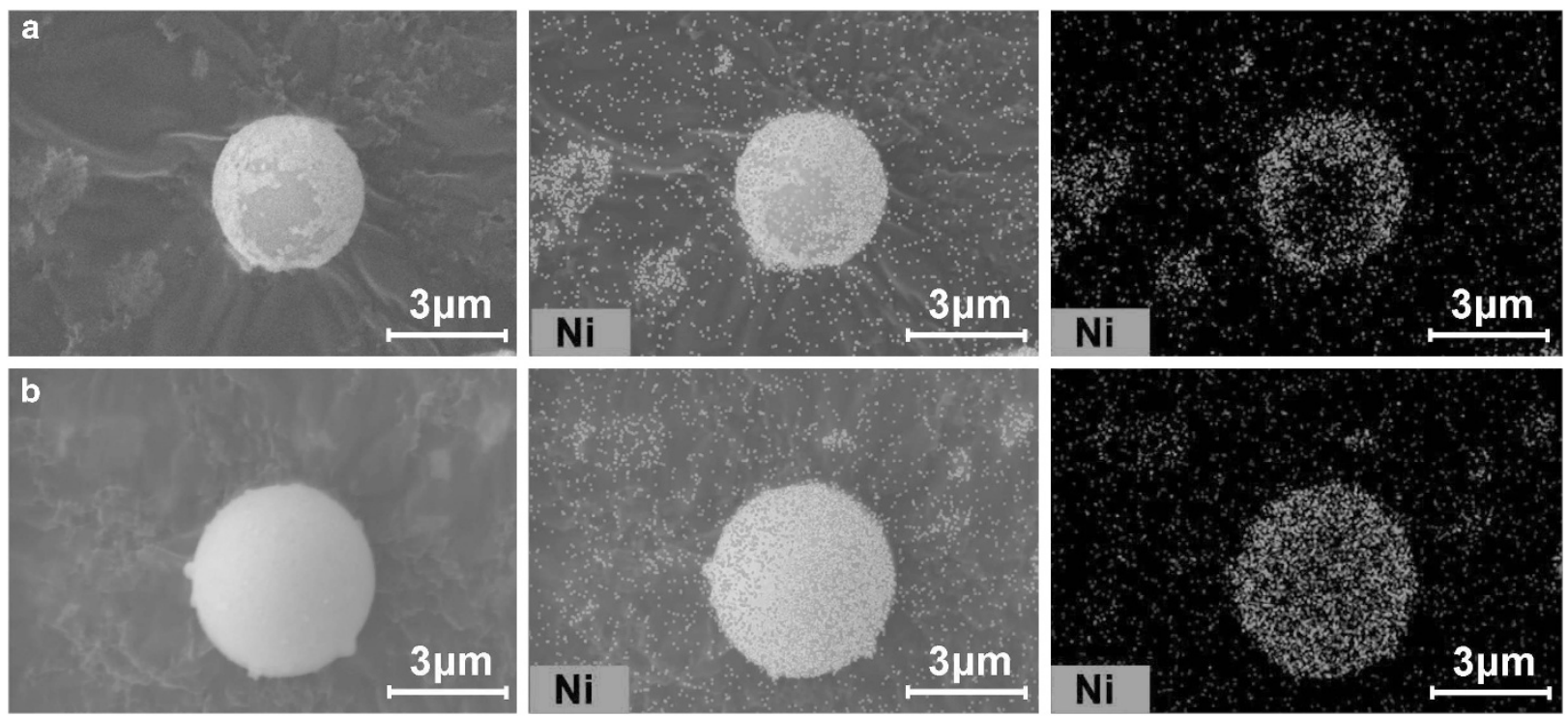

Figure 9 Element maps of EDX signals of $\mathrm{Ni}$ for (a) PS/Ni and (b) PSS4/Ni after ball mill durability tests for $0.5 \mathrm{~h}$. A full color version of this figure is available at Polymer Journal online.

microspheres as shown in Figure 6. The hollow structure was clearly observed in the broken microspheres, indicating that the PS cores had been completely removed. Figure 6 shows an important feature in the SEM images. The white wheel rim (nickel shell) around the microspheres is continuous and uniform, and the thickness of the observed nickel shell can be measured and the average thickness calculated. Figure 6 shows that (a) PS/Ni, (b) PSS1/Ni, (c) PSS2/Ni, (d) PSS3/Ni and (e) PSS4/Ni had nickel thicknesses of 132, 152, 165, 195 and $217 \mathrm{~nm}$, respectively. Figure 6a (PS/Ni) shows that the PS/nickel microspheres have a non-uniform layer with a nickel thickness of approximately $132 \mathrm{~nm}$. Figure 6e shows PSS4/Ni images of a continuous metal layer on aggregated nanoparticles, and the thickness is approximately $217 \mathrm{~nm}$. When the silane bridging agent concentrations increase, the PS microsphere surface has more $\mathrm{Si}-\mathrm{OH}$ function groups, which react with nickel ions to form $\mathrm{Si}-\mathrm{O}-\mathrm{Ni}$ covalent bonds and increase the thickness of the nickel layer. Therefore, the silane bridging agent concentration becomes the key factor in controlling the metal layer thickness. The concentration of the silane bridging agent (TSPM) can be used as a design parameter for PS microsphere nickel layer thickness.

Table 4 shows the $\mathrm{Ni}$ shell thickness obtained from different methods using a semi-quantitative approach. The nickel shell thickness of PS microsphere was calculated by using the results before and after electroless Ni-plating in Table 3 and by measuring the crosssections of the microspheres in the SEM images in Figure 6. The nickel shell thickness increases with the silane bridging agent content, and there are small differences between the two sets of data.

\section{XRD characterization}

Figure 7 shows the XRD patterns of PSS1 and PSS1/Ni microspheres. XRD verified the presence of nickel metal on the PS microspheres. The peaks that appeared at $2 \theta=44.5^{\circ}, 51.7^{\circ}$ and $75.7^{\circ}$ are attributed to the (lllll 111 , ( $\left.\begin{array}{lll}2 & 0 & 0\end{array}\right)$ and ( $\left.\begin{array}{lll}2 & 2 & 2\end{array}\right)$ planes of nickel, respectively. ${ }^{37}$ It can be concluded that a nickel coating forms on the surface of the PS microspheres during electroless plating.

\section{Mechanical stability}

Good adhesion between the plated Ni layer and the PS microspheres is important in preparing high-conductivity composites, such as antistatic and Anisotropic Conductive Film. ${ }^{38,39}$ Figures 8 a and e show the morphologies of Ni-plated PS microspheres with $0-1$ wt $\%$ TSPM, as determined by SEM, after the ball mill (for the same samples as in Figure 5). After $30 \mathrm{~min}$ of the ball mill, the PSS4/Ni microspheres remained intact, while the PS/Ni microspheres did not maintain their integrity. Figure 9 compares the element maps of EDX signals of $\mathrm{Ni}$ for (a) PS/Ni and (b) PSS4/Ni after the ball mill test for $30 \mathrm{~min}$ and demonstrates that $\mathrm{Ni}$ appears on the surfaces of the PS microspheres (shown by the red dots). There is more $\mathrm{Ni}$ metal and therefore stronger EDX signals of $\mathrm{Ni}$ in the element maps at high TSPM concentrations. These results indicate that PSS4/Ni contains sufficient silane bridging agent to promote good adhesion of nickel to the surfaces of the PS microspheres. Each $\mathrm{Si}$ atom of the silane bridging agent has three $-\mathrm{OH}$ groups; thus, the silane bridging agent can provide more bridging sites for combining with $\mathrm{Ni}$. The two-dimensional Si-O-Si network structure on the surface of the PS microspheres may provide another mechanism to improve the mechanical properties of these microspheres. The silane bridging agent makes the nickel layer surface of PS microspheres more compact and uniform in distribution and seems to provide a stronger bond between the metal shell and the polymer core, as well as more adsorption area for Si-O-Ni. PSS4/Ni has better mechanical stability than the other microspheres in the series in the ball mill and can be applied to conductive polymer composites.

\section{CONCLUSIONS}

Functionalized PS microspheres containing TSPM were prepared by dispersion copolymerization, and nickel layers were successfully introduced onto the surface of the microspheres by an electroless plating method. TSPM provided strong bonding ( $\mathrm{Si}-\mathrm{O}-\mathrm{Ni}$ ) between functionalized PS microspheres and nickel, forming a close and uniform metal layer on the functionalized PS microspheres. In particular, when the TSPM concentration was $1.0 \mathrm{wt} \%$, the PS microspheres remained intact, and the enhanced bridging efficiency 
provided the best performance in the ball mill. The enhancement of bridging efficiency increased as the TSPM concentration increased from 0.3 to $1.0 \mathrm{wt} \%$.

\section{CONFLICT OF INTEREST}

The authors declare no conflict of interest.

1 Lu, X., Zhang, H., Ni, Y., Zhang, Q. \& Chen, J. Porous nanosheet-based ZnO microspheres for the construction of direct electrochemical biosensors. Biosens. Bioelectron. 24, 93-98 (2008).

2 Kim, K., Lee, H. B., Park, H. K. \& Shin, K. S. Easy deposition of Ag onto polystyrene beads for developing surface-enhanced-Raman-scattering-based molecular sensors. J. Colloid Interface Sci. 318, 195-201 (2008).

3 Li, Y., Wang, Z., Gu, H. \& Xue, G. A facile strategy for synthesis of multilayer and conductive organo-silica/polystyrene/polyaniline composite particles. J. Colloid Interface Sci. 355, 269-273 (2011)

4 Wang, Y. \& Pan, C. Dielectric behavior and magnetic properties of poly(styrene-coacrylic acid)metal microspheres. Eur. Polym. J. 37, 699-704 (2001).

$5 \mathrm{Ma}, \mathrm{Y}$. \& Zhang, Q. Preparation and characterization of monodispersed PS/Ag composite microspheres through modified electroless plating. Appl. Surf. Sci. 258 7774-7780 (2012)

6 Wang, C., Zhang, C., Li, Y., Chen, Y. \& Tong, Z. Facile fabrication of nanocomposite microspheres with polymer cores and magnetic shells by Pickering suspension polymerization. React. Funct. Polym. 69, 750-754 (2009).

7 Islam, M. S., Yeum, J. H. \& Das, A. K. Synthesis of poly(vinyl acetate-methyl methacrylate) copolymer microspheres using suspension polymerization. J. Colloid Interface Sci. 368, 400-405 (2012).

8 Yin, D., Zhang, Q., Yin, C., Jia, Y. \& Zhang, H. Effect of particle coverage on morphology of $\mathrm{SiO}_{2}$-covered polymer microspheres by Pickering emulsion polymerization. Colloids Surf. A Physicochem. Eng. Asp. 367, 70-75 (2010).

9 Lee, J., Ha, J. U., Choe, S., Lee, C.-S. \& Shim, S. E. Synthesis of highly monodisperse polystyrene microspheres via dispersion polymerization using an amphoteric initiator. J. Colloid Interface Sci. 298, 663-671 (2006).

10 Zhu, Z., Sun, F., Yang, L., Gu, K. \& Li, W. Poly (styrene-co-maleic anhydride) microspheres prepared in ethanol/water using a photochemical method and their application in $\mathrm{Ni}^{2+}$ adsorption. Chem. Eng. J. 223, 395-401 (2013).

11 Kwon, S. H., Liu, Y. D. \& Choi, H. J. Monodisperse poly(2-methylaniline) coated polystyrene core-shell microspheres fabricated by controlled releasing process and their electrorheological stimuli-response under electric fields. J. Colloid Interface Sci. 440, 9-15 (2015).

12 Lee, J. H., Oh, J. S., Lee, P. C., Kim, D. O., Lee, Y. \& Nam, J. D. Fabrication of nickel/ gold multilayered shells on polystyrene bead cores by sequential electroless deposition processes. J. Electron. Mater. 37, 1648-1652 (2008).

13 Jiang, J., Lu, H., Zhang, L. \& Xu, N. Preparation of monodisperse Ni/PS spheres and hollow nickel spheres by ultrasonic electroless plating. Surf. Coat. Technol. 201, 7174-7179 (2007).

14 Rong, J., Yunwang, Z., Lin, Z., Chengfu, W. \& Jianjun, G. Study of electrodepositing Au on hollow polystyrene microspheres. Appl. Surf. Sci. 265, 465-469 (2013).

15 Lin, K. J., Wu, H. M., Yu, Y. H., Ho, C. Y., Wei, M. H., Lu, F. H. \& Tseng, W. J. Preparation of PMMA-Ni core-shell composite particles by electroless plating on polyelectrolyte-modified PMMA beads. Appl. Surf. Sci. 282, 741-745 (2013).

16 Chen, Y.-C., Liu, R. L.-H., Chen, X.-L., Shu, H.-J. \& Ger, M.-D. Microwave-assisted activation for electroless nickel plating on PMMA microspheres. Appl. Surf. Sci 257, 6734-6740 (2011).

$17 \mathrm{Wu}, \mathrm{X}, \mathrm{Qi}, \mathrm{S}$. \& Duan, G. Polyaniline/graphite nanosheet, polyaniline/Ag/graphite nanosheet, polyaniline/Ni/graphite nanosheet composites and their electromagnetic properties. Synth. Met. 162, 1609-1614 (2012).
18 Gao, D. L. \& Zhan, M. S. Fabrication and electrical properties of metal-coated acrylate rubber microspheres by electroless plating. Appl. Surf. Sci. 255 4185-4191 (2009).

19 Zhao, Y., Luo, W., Kanda, P., Cheng, H., Chen, Y., Wang, S. \& Huan, S. Silver deposited polystyrene (PS) microspheres for surface-enhanced Raman spectroscopicencoding and rapid label-free detection of melamine in milk powder. Talanta 113, 7-13 (2013).

20 Chao, Z., Wang, L., Song, L., Zhou, Y., Nie, W. \& Chen, P. Facile method to prepare poly (S-Co-HEA)/Ag nanocomposite particles with high efficient catalytic activity and surface enhanced Raman scattering. Appl. Surf. Sci. 329, 158-164 (2015).

21 Lee, J. H., Lee, Y. \& Nam, J. D. Tunable surface metal morphologies and electrical properties of monodispersed polystyrene beads coated with metal multilayers via electroless deposition. Intermetallics 17, 365-369 (2009).

$22 \mathrm{Lu}, \mathrm{Y}$. Electroless copper plating on 3-mercaptopropyltriethoxysilane modified PET fabric challenged by ultrasonic washing. Appl. Surf. Sci. 255, 8430-8434 (2009).

23 Liaw, W. C., Huang, P. C., Chen, K. P. \& Chen, C. S. Silane-bridged electroless Ni-plating on submicron polymer spheres. Polym. J. 41, 634-642 (2009).

$24 \mathrm{Lu}$, X. \& Xin, Z. Synthesis of poly(styrene-co-3-trimethoxysilyl propyl methacrylate) microspheres coated with polysiloxane layer. Colloid. Polym. Sci. 285, 599-604 (2007).

25 Xu, Y., Wen, Y., Wu, Y.-n., Lin, C. \& Li, G. Hybrid nanofibrous mats with remarkable solvent and temperature resistance produced by electrospinning technique. Mater. Lett. 78, 139-142 (2012).

26 Kim, M. S., Lee, G. H., Hong, J.-M. \& Lee, H. Synthesis of monodisperse PS-co-PDMS microspheres by dispersion polymerization. Mater. Sci. Eng. C 27, 1247-1251 (2007).

27 Graziola, F., Girardi, F., Di Maggio, R., Callone, E., Miorin, E., Negri, M., Müller, K. \& Gross, S. Three-components organic-inorganic hybrid materials as protective coatings for wood: Optimisation, synthesis, and characterisation. Prog. Org. Coat. 74, 479-490 (2012).

28 Mohseni, M., Bastani, S. \& Jannesari, A. Influence of silane structure on curing behavior and surface properties of sol-gel based UV-curable organic-inorganic hybrid coatings. Prog. Org. Coat. 77, 1191-1199 (2014).

29 Mimura, S., Naito, H., Kanemitsu, Y., Matsukawa, K. \& Inoue, H. Optical properties of organic-inorganic hybrid thin films containing polysilane segments prepared from polysilane-methacrylate copolymers. J. Organomet. Chem. 611, 40-44 (2000).

30 Rong, M. Z., Ji, Q. L., Zhang, M. Q. \& Friedrich, K. Graft polymerization of viny monomers onto nanosized alumina particles. Eur. Polym. J. 38, 1573-1582 (2002).

31 Smuleac, V., Butterfield, D. A., Sikdar, S. K., Varma, R. S. \& Bhattacharyya, D. Polythiol-functionalized alumina membranes for mercury capture. J. Membrane Sci. 251, 169-178 (2005)

32 Posthumus, W., Magusin, P. C. M. M., Brokken-Zijp, J. C. M., Tinnemans, A. H. A. \& van der Linde, R. Surface modification of oxidic nanoparticles using 3-methacryloxypropyltrimethoxysilane. J. Colloid Interface Sci. 269, 109-116 (2004).

$33 \mathrm{Ni}$, K. F., Shan, G. R., Weng, Z. X., Sheibat-Othman, N., Fevotte, G., Lefebvre, F. \& Bourgeat-Lami, E. Synthesis of hybrid core-shell nanoparticles by emulsion (co) polymerization of styrene and $\gamma$-Methacryloxypropyltrimethoxysilane. Macromolecules 38, 7321-7329 (2005).

34 Fan, X., Zheng, L., Cheng, J., Xu, S., Wen, X., Cai, Z., Pi, P. \& Yang, Z. Template synthesis of raspberry-like polystyrene/SiO2 composite microspheres and their application in wettability gradient surfaces. Surf. Coat. Technol. 213, 90-97 (2012).

35 Hsiue, G.-H., Kuo, W.-J., Huang, Y. -P. \& Jeng, R.-J. Microstructural and morphological characteristics of PS-SiO 2 nanocomposites. Polymer 41, 2813-2825 (2000).

$36 \mathrm{Lu}$, X. \& Xin, Z. Preparation and characterization of micron-sized polystyrene/ polysiloxane core/shell particles. Colloid. Polym. Sci. 284, 1062-1066 (2006).

37 Song, D., Zhou, J., Jiang, W., Zhang, X., Yan, Y. \& Li, F. A novel activation for electroless plating on preparing Ni/PS microspheres. Mater. Lett. 63, 282-284 (2009).

$38 \mathrm{He}$, J., Chen, Q. Huang, H., Zheng, L., Chen, B. \& Lin, J. Development of novel anisotropic Janus composite particles based on Urushiol-iron/polystyrene polymer. Prog. Org. Coat. 85, 15-21 (2015).

39 Pang, H., Xu, L., Yan, D.-X. \& Li, Z.-M. Conductive polymer composites with segregated structures. Prog. Polym. Sci. 39, 1908-1933 (2014). 